\title{
General Lower Bounds for the Minor Crossing Number of Graphs
}

\author{
Drago Bokal · Éva Czabarka • László A. Székely • \\ Imrich Vrt'o
}

Received: 17 June 2008 / Revised: 16 September 2009 / Accepted: 20 January 2010 /

Published online: 17 June 2010

(C) Springer Science+Business Media, LLC 2010

\begin{abstract}
There are three general lower bound techniques for the crossing numbers of graphs: the Crossing Lemma, the bisection method and the embedding method. In this contribution, we present their adaptations to the minor crossing number. Using the adapted bounds, we improve on the known bounds on the minor crossing number of hypercubes. We also point out relations of the minor crossing number to string graphs and establish a lower bound for the standard crossing number in terms of Randič index.
\end{abstract}

Keywords Minor crossing number · Crossing number · Graph minor · Hypercube · String graphs

D. Bokal was supported in part by the Ministry of Higher Education, Science and Technology of Slovenia, Research Project J1-2043 and Research Program P1-0297.

L.A. Székely was supported in part by the NSF DMS contracts 0701111 and 1000475.

É. Czabarka was supported in part by a 2010 PIRA track I internal grant of the University of South Carolina.

É. Czabarka, L.A. Székely and I. Vrt'o were supported by the EPSRC grant GR/S76694/01.

I. Vrt'o was also partially supported by the Vega grant No. 2/0111/09 and the APVV grant No. 0433/06.

D. Bokal $(\bowtie)$

Faculty of Natural Sciences and Mathematics, University of Maribor, Koroška 160, 2000 Maribor, Slovenia

e-mail: drago.bokal@uni-mb.si

É. Czabarka · L.A. Székely

Department of Mathematics, University of South Carolina, Columbia, SC 29208, USA

I. Vrt'o

Institute of Mathematics, Slovak Academy of Sciences, Dúbravská 9, 84104 Bratislava,

Slovak Republic 


\section{Preliminaries}

The minor crossing number of a graph $G$ on a surface $\Sigma$, introduced in [6], is defined as the minimum crossing number of all graphs that contain $G$ as a minor:

$$
\operatorname{mcr}(G, \Sigma):=\min \left\{\operatorname{cr}(H, \Sigma) \mid G \leq_{m} H\right\} .
$$

(As usual, the notation $G \leq_{m} H$ means that $G$ is a minor of $H$.) By $\operatorname{mcr}(G)$, we denote $\operatorname{mcr}\left(G, \mathbb{S}_{0}\right)$, the crossing number in the sphere $\mathbb{S}_{0}$.

For each graph $G$ and each surface $\Sigma$ there exists a realizing graph $\bar{G}$, such that $G \leq_{m} \bar{G}$ and $\operatorname{mcr}(G, \Sigma)=\operatorname{cr}(\bar{G}, \Sigma)$. An optimal drawing of $\bar{G}$ in $\Sigma$ is called a realizing drawing of $G$. We clearly may assume and shall do so from now on, that $G$ and $\bar{G}$ have the same number of connected components.

$G$ can be obtained as a contraction of a subgraph of $\bar{G}$. In other words, $G=$ $(\bar{G}-R) / C$ for suitable edge sets $R, C \subseteq E_{\bar{G}}$. The edges of $R$ are called the removed edges and those in $C$ are the contracted edges. Note that the edge-set $C$ is acyclic and that $E_{G}=E_{\bar{G}} \backslash(R \cup C)$ are the original edges of $G$. It is clear that every graph $G$ has a realizing graph $\bar{G}$ such that $R=\emptyset$. A stronger claim can be established using the following theorem, cf. Lemma 2. (Recall that the Euler genus of an (orientable or nonorientable) surface $\Sigma$ is defined as $g(\Sigma)=2-\chi(\Sigma)$, where $\chi(\Sigma)$ is the Euler characteristic of the surface. So the Euler genus of an orientable surface with $k$ handles is $2 k$, and the Euler genus of a nonorientable surface with $k$ crosscaps is $k$.)

As precise definitions of the concepts we use in this paper are quite lengthy, we omit several basic definitions. Those about crossing numbers are well addressed in [6], and [19] is an excellent reference for graph embeddings in general (nonorientable) surfaces.

According to [6], it has long been lamented that the (ordinary) crossing number does not work well with graph minors, as it is not a minor monotone invariant. This can be overcome by the above minor crossing number, which allows for additional degrees of freedom in drawing graphs: instead of drawing vertices as points and edges as curves, a vertex of the original graph can be represented by a tree in a realizing drawing. In such drawing, the edges of these trees can intersect, which corresponds to the possibility that vertices intersect each other in the representation of the original graph. These additional degrees of freedom allow for further minimizing the number of crossings. Such an approach is particularly useful in the original application of crossing numbers, VLSI circuit design, where we can minimize the number of crossings needed for a specific circuit by expanding high-degree vertices into trees, such that each vertex of the tree has the same electric potential in the circuit.

It is easy to see that there are graphs with arbitrary difference between ordinary and minor crossing number. In [6], the authors show that the minor crossing number of $K_{n}$ is $\Theta\left(n^{2}\right)$, which is orders of magnitude lower than the crossing number of $K_{n}$, which is $\Theta\left(n^{4}\right)$. In the drawings that establish the upper bound on the minor crossing number, there are no crossings of the original edges of $K_{n}$, and almost every pair of vertex-trees cross. This example also shows that ordinary crossing number bounds do not apply to the minor crossing number.

Theorem 1 ([6], Theorem 5.4) Let $\Sigma$ be a surface of Euler genus $g$ and let $k \geq 1$ be an integer. Then $\operatorname{mcr}(G, \Sigma) \leq k$ holds for a graph $G$, if and only if $G$ can be 
embedded in the nonorientable surface $\mathbb{N}_{g+k}$ of Euler genus $g+k$, so that there exist pairwise noncrossing onesided curves $\gamma_{1}, \ldots, \gamma_{k}$ in $\mathbb{N}_{g+k}$, each of which intersects the embedding of $G$ in at most two points, and such that we obtain $\Sigma$ by pasting disks into $\mathbb{N}_{g+k}$ along those curves.

Lemma 2 Let $G$ be a graph and $\Sigma$ a surface. There exists a realizing drawing $\bar{D}$ of some realizing graph $\bar{G}$ of $G$ in $\Sigma$, such that (i) $|V(\bar{G})|-|V(G)|=|E(\bar{G})|-$ $|E(G)| \leq 2 \operatorname{mcr}(G, \Sigma)$, (ii) the crossings in $\bar{D}$ involve only contracted edges of $\bar{G}$, and (iii) no two edges of $\bar{G}$ that are contracted to the same vertex of $G$ cross in $\bar{D}$.

Proof Let $\Sigma$ have Euler genus $g$, let $k=\operatorname{mcr}(G, \Sigma)$, and let $\gamma_{1}, \ldots, \gamma_{k}$ be the curves guaranteed by Theorem 1. By moving the curves along the edges, we may assume that the curves intersect the embedding $D$ of $G$ in $\mathbb{N}_{g+k}$ in the vertices of $G$ only.

Let $\Delta_{1}, \ldots, \Delta_{k}$ be the disks we paste along $\gamma_{1}, \ldots, \gamma_{k}$ into $\mathbb{N}_{g+k}$ to obtain $\Sigma$. Each disk has at most two points of $G$ on its boundary, which correspond to four intertwined points in the cut surface. By adding (at most) two edges and precisely one new vertex for every new edge, and by drawing them as paths on the corresponding disk, we obtain a drawing $\bar{D}$ of a graph $\bar{G}$ in $\Sigma$, which has $G$ as a minor. In each disk $\Delta_{i}$, we introduced at most two new vertices, two new edges and one crossing. As $G$ was embedded in $\mathbb{N}_{g+k}$ with no crossings, the drawing satisfies (i) and (ii).

Suppose one of the crossings would be a crossing of two edges that are contracted to the same vertex of $G$. We can replace this crossing by a vertex: the new graph still has $G$ as a minor, but its drawing in $\Sigma$ has fewer than $\operatorname{mcr}(G, \Sigma)$ crossings. The contradiction implies there are no such crossings, thus $\bar{D}$ satisfies (iii).

The main motivation for this paper is to examine how do the general lower bounds for the ordinary crossing number translate to the minor crossing number. The most difficult challenge to overcome is the problem of studying the ordinary crossing number of not just a single graph at a time, but of the whole family of graphs that can be obtained from the given graph by replacing its vertices by trees in arbitrary fashion. The tools for ordinary crossing number clearly do not adequately address this challenge. We organize our approach to this challenge as follows: in Sect. 2 we combine the probabilistic method with Randič index to produce a crossing-lemma-like bound on the minor crossing number, in Sect. 3 we introduce variants of bisection width that are adapted to studying bisections in the family of graphs containing a specific minor and produce a bisection width-based lower bound on the minor crossing number, and in Sect. 4 we refine the embedding method in order to provide a lower bound on the minor crossing number of graphs that is both an extension of the embedding method $[17,27]$ to the minor crossing number on one side as well as a generalization of results of Garcia-Moreno and Salazar [10] and Bokal, Fijavž, and Mohar [6] on the other side. We conclude with a section on applications, where we improve the bound on the minor crossing number of hypercubes from [6] and show an interesting link between minor crossing numbers and string graphs.

\section{The Crossing Lemma}

The following result was conjectured by Erdős and Guy [9] for a general constant $c$ in place of $\frac{1}{64}$, and then proven by Leighton [17] and independently by Ajtai, Chvá- 
tal, Newborn, and Szemerédi [2] for $c=\frac{1}{100}$. A folklore beautiful application of the probabilistic method derives it from the Euler Formula [1]. The sketch of the proof of Theorem 4 refers to this proof.

Theorem 3 (The Crossing Lemma, [2, 17]) Let $G$ be a graph of order $n$ with $m \geq 4 n$ edges. Then, $\operatorname{cr}(G) \geq \frac{1}{64} \frac{m^{3}}{n^{2}}$.

In order to asymptotically improve the Crossing Lemma, one needs to add some assumptions to the graphs (for example high girth, see [22]). However, the constant is far from tight and was improved several times. The current strongest version is by Pach, Radoičić, Tardos, and Tóth [23]. Székely found many applications in discrete geometry [31].

Recall the definition of the generalized Randič index of a connected graph $G$,

$$
R^{\alpha}(G)=\sum_{\{u, v\} \in E(G)} d_{u}^{\alpha} d_{v}^{\alpha},
$$

where $\alpha$ is a real number and $d_{v}$ denotes the degree of the vertex $v$. Further, we use $\Delta(G)$ and $\delta(G)$ for the largest and the smallest degree of a vertex in $G$, respectively. Somewhat intriguingly, this quantity, coming from mathematical chemistry, is involved in a lower bound for the minor crossing number of graphs:

Theorem 4 Let $G$ be a simple graph without isolated vertices that has girth $r$ and let $\Sigma$ be a surface of Euler genus $g$ and $\alpha \in \mathbb{R}$. For $\alpha \geq 0$, define $d_{\alpha}=\Delta(G)$, and let $d_{\alpha}=\delta(G)$ otherwise. Then, the following holds:

$$
\operatorname{mcr}(G, \Sigma) \geq \frac{r-2}{r+2} \frac{R^{\alpha}(G)}{d_{\alpha}^{2 \alpha}}-\frac{r}{r+2}\left(\sum_{v \in V(G)}\left(\frac{d_{v}}{d_{\alpha}}\right)^{\alpha}+g\right) .
$$

Proof Assume that $\bar{G}$ is a realizing graph of $G$ in $\Sigma$, i.e. $\operatorname{mcr}(G, \Sigma)=\operatorname{cr}(\bar{G}, \Sigma)$. By Lemma 2 we may assume that a vertex $v$ of $G$ has been substituted by $i_{v}+1$ vertices (of which $i_{v}$ are new vertices) and $i_{v}$ edges. Apply the Crossing Lemma proof to $\bar{G}$, such that we make independent experiments for the $i_{v}+1$-tuples of vertices (i.e. include all or none) with probability $\left(d_{v}(G) / d_{\alpha}\right)^{\alpha} \leq 1$. Note that unlike in the proof of the Crossing Lemma, the probability distribution is no longer uniform.

By Lemma 2, we can simultaneously assume three properties on $\bar{G}$ and its drawing $\bar{D}$ :

(i) $\sum_{v \in V(G)} i_{v} \leq 2 \operatorname{mcr}(G, \Sigma)$,

(ii) the crossings in $\bar{G}$ involve only contracted edges of $\bar{G}$, and

(iii) edges that are contracted to the same vertex $v \in V(G)$ do not cross each other in $\bar{D}$.

Let $e_{v}$ be an edge that is contracted to the vertex $v \in V(G)$. Then,

$$
\operatorname{mcr}(G, \Sigma)=\operatorname{cr}(\bar{G}, \Sigma) \geq \sum_{e_{u}, e_{v} \in E(\bar{G}) \text { cross }}\left(\frac{d_{u}}{d_{\alpha}}\right)^{\alpha}\left(\frac{d_{v}}{d_{\alpha}}\right)^{\alpha} .
$$


On the other hand, we take the random subgraph $G^{\prime}$ of $\bar{G}$ and the corresponding subdrawing of $\bar{D}$ by picking disjoint sets of vertices above with the prescribed probabilities. For any simple graph $H, \operatorname{cr}(H, \Sigma) \geq e(H)-\frac{r}{r-2}(n(H)+g)$ by the Euler Formula. We spell out this inequality for $G^{\prime}$ and take the expectation. The expected number of vertices in $G^{\prime}$ is $\sum_{v \in V(G)}\left(d_{v} / d_{\alpha}\right)^{\alpha}\left(i_{v}+1\right)$; the expected number of edges in $G^{\prime}$ is $R^{\alpha}(G) / d_{\alpha}^{2 \alpha}+\sum_{v \in V(G)}\left(d_{v} / d_{\alpha}\right)^{\alpha} i_{v}$ (corresponding to original and contracted edges), according to the linearity of expectation. Finally, the expected number of crossings of $G^{\prime}$ in $\bar{D}\left[G^{\prime}\right]$ is $\sum_{e_{u}, e_{v} \in E(\bar{G}) \text { cross }}\left(\frac{d_{u}}{d_{\alpha}}\right)^{\alpha}\left(\frac{d_{v}}{d_{\alpha}}\right)^{\alpha}$, which is greater than or equal to the expected crossing number of $G^{\prime}$. Combining these results with assumption (i), we obtain

$$
\begin{aligned}
& \sum_{e_{u}, e_{v} \in E(\bar{G}) \mathrm{cross}}\left(\frac{d_{u}}{d_{\alpha}}\right)^{\alpha}\left(\frac{d_{v}}{d_{\alpha}}\right)^{\alpha} \\
\geq & \frac{R^{\alpha}(G)}{d_{\alpha}^{2 \alpha}}+\sum_{v \in V(G)}\left(\frac{d_{v}}{d_{\alpha}}\right)^{\alpha} i_{v}-\frac{r}{r-2}\left(\sum_{v \in V(G)}\left(\frac{d_{v}}{d_{\alpha}}\right)^{\alpha}\left(i_{v}+1\right)+g\right) \\
\geq & \frac{R^{\alpha}(G)}{d_{\alpha}^{2 \alpha}}-\frac{r}{r-2}\left(\sum_{v \in V(G)}\left(\frac{d_{v}}{d_{\alpha}}\right)^{\alpha}+g\right)-\frac{4}{r-2} \operatorname{mcr}(G, \Sigma),
\end{aligned}
$$

which together with (2.1) implies the claim.

Combining the same non-uniform probability distribution with the proof of the ordinary Crossing Lemma, we obtain the following:

Theorem 5 Let $G$ be a simple graph of girth $r$ that has no isolated vertices. For $\alpha \geq 0$, define $d_{\alpha}=\Delta(G)$, and let $d_{\alpha}=\delta(G)$ otherwise. If $R^{\alpha}(G) \geq \frac{3}{2} \frac{r}{r-2} d_{\alpha}^{\alpha} \sum_{v \in V(G)} d_{v}^{\alpha}$, then the following holds:

$$
\operatorname{cr}(G) \geq \frac{4}{27} \frac{1}{d_{\alpha}^{4 \alpha}}\left(\frac{r-2}{r}\right)^{2} \frac{\left(R^{\alpha}(G)\right)^{3}}{\left(\sum_{v \in V(G)} d_{v}^{\alpha}\right)^{2}} .
$$

This theorem has little to say about girth in view of results of Pach, Spencer, and Toth [22]. However, we present it as a possible direction of research into combining the Crossing Lemma proofs with non-uniform probability distributions on graph vertices. (In this regard, we acknowledge interesting discussions with Lincoln Lu.)

Note that Theorem 4 simplifies to the bound implied by the Euler Formula in the case $\alpha=0$; in this case, all probabilities are equal to 1 . Similarly, Theorem 5 reduces to the bound of the Crossing Lemma. For $\alpha>0$, the bound favors vertices with large degree, and, for $\alpha<0$, it favors vertices with small degree. Note that $\alpha<0$ may give better results than $\alpha>0$ : an example is a graph $L(n, k)$ obtained from a disjoint union of $n$ copies of a complete graph $K_{k}$ and an independent set $\bar{K}_{k}$ by connecting vertices of complete graphs with corresponding vertices of the independent set, so that in the final graph, all vertices of the independent set have degree $n$ and all vertices of the complete graphs have degree $k$. Such behavior of the bound from Theorem 4 indicates 
that the edges incident with vertices of small degree contribute most of the crossings in the graph.

Like the Crossing Lemma, Theorems 4 and 5 can be applied without any knowledge of graph's global structure: the only information we need are the degrees of adjacent vertices. Contrary to the Crossing Lemma, however, Theorem 4 does not require any restrictions on the density of the graph. In fact, it performs best on sparse graphs that have a dense part. For Theorem 5, however, the edge-density condition is replaced by a bound on the generalized Randič index of the graph.

As an example, consider a graph $R(n, m)$, which is obtained by $m$-times subdividing each edge incident to a fixed vertex $v$ of $K_{n+1}$ and consistently connecting all vertices at a fixed distance $d=1,2, \ldots, m$ from $v$ in a cycle (more precisely, number the edges adjacent to $v$ by $1,2, \ldots, n$, and let $y_{j, i}$ be a subdivision point on edge $i$ at distance $j$ from $v$. Add the edges $y_{1, i} y_{2, i}, y_{2, i} y_{3, i}, \ldots, y_{m-1, i} y_{m, i}$ and $y_{m, i} y_{1, i}$ for each $i)$. Then $\operatorname{mcr}(R(n, m)) \leq \operatorname{cr}(R(n, m))=\operatorname{cr}\left(K_{n+1}\right)$, but the average degree of $R(n, m)$ is close to four for large $m$, thus, the nonstructural bounds considering just the number of edges and vertices become trivial (Euler bound, Crossing Lemma). Theorem 4, however, produces the following bound:

$$
\operatorname{mcr}(R(n, m)) \geq \frac{1}{10}\left(2 n(2-3 m)\left(\frac{4}{n}\right)^{\alpha}+2 n(2 m-1)\left(\frac{4}{n}\right)^{2 \alpha}+n^{2}-7 n-6\right) .
$$

For $n>4, m \geq 0$, and $\alpha \rightarrow \infty$, this expression simplifies to $\frac{1}{10}\left(n^{2}-7 n-6\right)$, which is approximately $\frac{2}{5}$ of the best known lower bound for $K_{n}$. A similar computation for the graphs $K_{m, n}$ produces $\frac{2}{3}$ of the best known lower bound for $\operatorname{mcr}\left(K_{m, n}\right)$, applying $\alpha=0$. Even non-integer values of $\alpha$ can give best results. For instance, the graph $10 R(199,100)+10\left(K_{100} * K_{100}^{c}\right)$ has largest bound at $\alpha \approx 1.032\left(G^{c}\right.$ is the complement of $G, G+H$ is the disjoint union, and $G * H$ is the complete join of $G$ and $H$ ).

Similarly for the ordinary crossing number, the edge density condition of the Crossing Lemma is violated for graphs $R(n, m)$ with large $m$, but with $\alpha \rightarrow \infty$, the value of generalized Randič index satisfies the condition of Theorem 5, which thus yields a lower bound of the same order of magnitude and with the same constant factor as the Crossing Lemma. Again, the graph $10 R(199,100)+10\left(K_{100} * K_{100}^{c}\right)$ is an example with a non-integer optimal $\alpha \approx 2.247$.

\section{The Bisection Method}

In 1982, Leighton was interested in bounded-degree graphs for VLSI design [17]. He invented the bisection method and showed that $\operatorname{cr}(G)+n$ is bounded from below by the squared bisection width of the graph multiplied by a small constant (the bisection width parallels the concepts defined below). This result was later extended to general graphs by Pach, Shahrokhi and Szegedy [21], who also produced specific constants and replaced $n$ with the sum of degree squares. Independently, Sýkora and Vrt'o [30] proved an essentially equivalent result for crossing numbers of general graphs in surfaces of higher genus. One might say that this is the single most powerful technique to 
set bounds on the crossing number. All bisection width techniques depend on some (planar or otherwise) separator theorem. We generalize this technique to the minor crossing number almost directly. As the reader readily can see, the vertices and edges swap roles in this bisection width generalization to the minor crossing number. As shown in Lemma 7, the minimum possible edge bisection width of a realizing graph is tightly estimated by the strong vertex bisection width. Consequently, the method is perhaps most strong (and probably easiest to use) in regular graphs. We give an application for such a regular graph, the $n$-dimensional hypercube, later in this paper.

As usual, for $X, Y \subseteq V(G)$, by $\langle X\rangle$ we denote the subgraph of $G$ spanned by $X$, by $E(X, Y)$ we denote the set of edges $x y \in E(G)$ such that $x \in X$ and $y \in Y$, and $E(X)=E(X, X)$.

Let $G$ be a graph, $\alpha \in(0,1 / 2]$, and $W \subseteq V(G)$. A set of edges $F \subseteq E(G)$ is an $\alpha$-edge bisection of the vertices of $G$ with respect to $W$ (in short, $\alpha$-edge bisection of $G$ with respect to $W)$, if $V(G)$ can be partitioned into $V_{1}$ and $V_{2}$ such that $\min \left(\mid V_{1} \cap\right.$ $\left.W|,| V_{2} \cap W \mid\right) \geq \min \left(\lceil\alpha|W|\rceil,\left\lfloor\frac{1}{2}|W|\right\rfloor\right)$, and every edge between $V_{1}$ and $V_{2}$ in $G$ belongs to $F$. We denote by $\operatorname{bw}_{e}(G, W ; \alpha)$ the size of the smallest $\alpha$-edge bisection of $G$ with respect to $W$, and use $\operatorname{bw}_{e}(G, W)$ if $\alpha=1 / 2$.

Note that $|W| \geq 2$ implies that $V_{i} \cap W \neq \emptyset$ for $i \in\{1,2\}$. The condition $\min \left(\left|V_{1} \cap W\right|,\left|V_{2} \cap W\right|\right) \geq \min \left(\lceil\alpha|W|\rceil,\left\lfloor\frac{1}{2}|W|\right\rfloor\right)$ is equivalent with $\max \left(\mid V_{1} \cap\right.$ $\left.W|,| V_{2} \cap W \mid\right) \leq \max \left(\lfloor(1-\alpha)|W|\rfloor,\left\lceil\frac{1}{2}|W|\right\rceil\right)$. If $\alpha \leq \frac{1}{2}-\frac{1}{2|W|}$ or $|W|$ is even, then $\min \left(\lceil\alpha|W|\rceil,\left\lfloor\frac{1}{2}|W|\right\rfloor\right)=\lceil\alpha|W|\rceil$, so we can replace the condition on $\left|V_{i} \cap W\right|$ with $\min \left(\left|V_{1} \cap W\right|,\left|V_{2} \cap W\right|\right) \geq \alpha|W|$, as one would expect. However, if $|W|$ is odd and $\alpha \in\left(\frac{1}{2}-\frac{1}{2|W|}, \frac{1}{2}\right]$, then the condition $\min \left(\left|V_{1} \cap W\right|,\left|V_{2} \cap W\right|\right) \geq$ $\min \left(\lceil\alpha|W|\rceil,\left\lfloor\frac{1}{2}|W|\right\rfloor\right)$ is equivalent with $\min \left(\left|V_{1} \cap W\right|,\left|V_{2} \cap W\right|\right)=\left\lfloor\frac{1}{2}|W|\right\rfloor<\alpha|W|$.

Although it may seem at first glance that the ceilings and floors in the above definition are not necessary, as our note in the previous paragraph shows, they turn essential in exact calculations when $|W|$ is odd. For different reasons that should be easily discernible to the attentive reader, such finery is unnecessary in the definitions that follow.

A set $S \subseteq V$ is an $\alpha$-vertex bisection of the edges of $G$ (in short, an $\alpha$-vertex bisection of $G$ ), if the vertices of $G-S$ are partitioned into sets $V_{1}$ and $V_{2}$, such that the graph induced by $V_{i}, i=1,2$, has at most $(1-\alpha)|E|$ edges; if $E(G) \neq \emptyset$, then we require that, for $i=1,2, E\left(V_{i} \cup S\right) \neq \emptyset$ and that there is no edge between $V_{1}$ and $V_{2}$ in $G$. Let $\operatorname{bw}_{v}(G ; \alpha)$ denote the cardinality of the smallest $\alpha$-vertex bisection of $G$; again we use $\operatorname{bw}_{v}(G)$ if $\alpha=1 / 2$.

A set $S \subseteq V$ is a strong $\alpha$-vertex bisection of the edges of $G$ (in short, a strong $\alpha$ vertex bisection of $G)$, if the vertices of $G-S$ can be partitioned into two sets $V_{1}$ and $V_{2}$ such that $\min \left(E\left(V_{1} \cup S\right), E\left(V_{2} \cup S\right)\right) \geq \alpha|E(G)|$, and there is no edge between $V_{1}$ and $V_{2}$ in $G$. Let $\mathrm{bw}_{v}^{*}(G ; \alpha)$ denote the cardinality of the smallest strong $\alpha$-vertex bisection of $G$, again $\alpha=1 / 2$ is simply omitted from the notation.

Lemma 6 Let $\alpha \in(0,1 / 2]$. For any $G=(V, E), \operatorname{bw}_{v}^{*}(G ; \alpha) \geq \operatorname{bw}_{v}(G ; \alpha)$.

Proof The statement is trivial if $E(G)=\emptyset$, so assume $E(G) \neq \emptyset$. Let $S$ be a strong $\alpha$-vertex bisection of $G$. Then for $i \in\{1,2\}$ we have that $E\left(V_{i} \cup S\right) \neq \emptyset$ and $\left|E\left(V_{i}\right)\right| \leq$ 
$|E(G)|-\left|E\left(V_{3-i} \cup S\right)\right| \leq(1-\alpha)|E(G)|$, so $S$ is also an $\alpha$-vertex bisection of $G$ and the claim follows.

For a graph $G=(V, E)$, we define $\mathcal{H}_{G}$ as the set of all graphs $\bar{G}$ that can be obtained from $G$ as follows. For every vertex $v \in V$, subdivide every edge incident to $v$. Then each edge is subdivided twice. The new vertices are called leaves. If $v$ has degree at least three, remove $v$ and attach a tree to the leaves, so that the internal vertices of the tree are of degree three. Note that the number of internal tree vertices in every such tree equals the number of leaves minus two. The edges of these trees, as well as the edges incident to degree one or two vertices of the original $G$, are called tree edges of $\bar{G} \in \mathcal{H}$. The non-leaf tree vertices are internal vertices. Note that the vertices that have degree one in $G$ are also internal vertices. Let $\bar{G} \in \mathcal{H}_{G}$, then the number of internal tree vertices at every tree $T_{v}, v \in G$, equals at least the number of leaves less two (equality holds if the corresponding vertex $v$ in $G$ has degree at least three). For $\bar{G} \in \mathcal{H}_{G}$ we denote the set of leaves by $L$. Then $|L|=2|E|$. Let $I$ be the set of all internal vertices of trees, then $|I| \geq|L|-2|V|=2|E|-2|V|$ (with equality if the minimum degree of $G$ is 3 ). The newly introduced concepts are related in Lemma 7.

Lemma 7 Let $\alpha \in(0,1 / 2]$. For any $G=(V, E)$ without isolated vertices,

$$
\mathrm{bw}_{v}^{*}(G ; \alpha) \geq \min _{\bar{G} \in \mathcal{H}_{G}} \mathrm{bw}_{e}(\bar{G}, L ; \alpha) \geq \mathrm{bw}_{v}^{*}(G ; \alpha)-1 \geq \mathrm{bw}_{v}(G ; \alpha)-1 .
$$

Proof Again, if $E=\emptyset$, the statement is trivial, so let $G$ be a graph such that $E \neq \emptyset$, so $|L|=2|E| \geq 2$. By Lemma 6 it is enough to prove that $\mathrm{bw}_{v}^{*}(G ; \alpha) \geq$ $\min _{\bar{G} \in \mathcal{H}_{G}} \operatorname{bw}_{e}(\bar{G}, L ; \alpha) \geq \mathrm{bw}_{v}^{*}(G ; \alpha)-1$.

Let $\bar{G} \in \mathcal{H}_{G}$ be a graph that has minimal $\alpha$-edge bisection width with respect to $L$, and let $F$ be the smallest $\alpha$-edge bisection of $\bar{G}$ with respect to $L$, i.e., $|F|=$ $\operatorname{bw}_{e}(\bar{G}, L ; \alpha)$. Since $|L|=2|E|$ is even, the graph $\bar{G}-F$ consists of subgraphs $\bar{G}_{1}$, $\bar{G}_{2}$ both having at least $\lceil\alpha|L|\rceil>0$ leaves.

Any edge $e \in F$ not belonging to any of the trees that replaced the vertices of $G$ in $\bar{G}$ is adjacent with precisely one tree edge $e_{1}$ in $\bar{G}_{1}$ and another tree edge $e_{2}$ in $\bar{G}_{2}$. We obtain a set $F^{\prime}$ by replacing every such $e \in F$ with one of $e_{i}$, such that the sides are chosen evenly; then at most one non-tree edge $e$ is left in $F^{\prime}$, and $|F|=\left|F^{\prime}\right|$. The set $F^{\prime}$ separates $\bar{G}$ into $\bar{G}_{1}^{\prime}$ and $\bar{G}_{2}^{\prime}$. Since every leaf in $\bar{G}_{i}^{\prime}$ is incident to a single non-tree edge in $E\left(\bar{G}_{i}^{\prime}\right) \cup\{e\}$, and every non-tree edge is incident upon precisely two leaves, the number of non-tree edges in $E\left(\bar{G}_{i}^{\prime}\right) \cup\{e\}$ is at least $\frac{\alpha|L|}{2}=\alpha|E|$. By contracting every tree of $\bar{G}$ to a vertex we get the original graph $G$. For $i=1,2$, let $G_{i}$ be a subgraph of $G$ spanned by the vertices that were contracted from trees containing leafs from $\bar{G}_{i}^{\prime}$. Note that $G_{1}$ and $G_{2}$ share vertices that correspond to trees containing edges from $F^{\prime}$. Then $E\left(G_{i}\right)$ contains the non-tree edges of $E\left(\bar{G}_{i}^{\prime}\right) \cup\{e\}$, and so $\left|E\left(G_{i}^{*}\right)\right| \geq \alpha|E|$. Let $S$ be the set of vertices in $G$ that correspond to trees containing edges from $F^{\prime}$ and the endvertices of $e$. Clearly, the vertices of $G-S$ can be partitioned into $V_{1}$ and $V_{2}$ such that $V_{i} \subseteq V\left(G_{i}\right) \subseteq V_{i} \cup S$ and every path from $V_{1}$ to $V_{2}$ goes through a vertex of $S$. Also, $\left|E\left(S \cup V_{i}\right)\right| \geq\left|E\left(G_{i}\right)\right| \geq \alpha|E|$. Then $S$ is a 
strong $\alpha$-vertex bisection of $G$ of size at most $|F|+1$ (and the edge $e$ is in $\left\langle V_{i} \cup S\right\rangle$ for both $i=1,2)$. Hence we proved the second inequality:

$$
\operatorname{bw}_{e}(\bar{G}, L ; \alpha)=|F| \geq|S|-1 \geq \mathrm{bw}_{v}^{*}(G ; \alpha)-1 \text {. }
$$

For the first inequality, let $S \subseteq V$ be a strong $\alpha$-vertex bisection in $G$ such that $|S|=\operatorname{bw}_{v}^{*}(G ; \alpha)$, and let $V_{1}, V_{2} \subseteq V$ be the partition of $G$ corresponding to $S$. Now either $\lceil\alpha|E|\rceil \leq\left\lfloor\frac{1}{2}|E|\right\rfloor$ or $\lceil\alpha|E|\rceil=\left\lceil\frac{1}{2}|E|\right\rceil>\left\lfloor\frac{1}{2}|E|\right\rfloor$. If $\lceil\alpha|E|\rceil \leq\left\lfloor\frac{1}{2}|E|\right\rfloor$, then let $C=\emptyset$. If $\lceil\alpha|E|\rceil=\left\lceil\frac{1}{2}|E|\right\rceil>\left\lfloor\frac{1}{2}|E|\right\rfloor$, then we must have $E(S) \neq \emptyset$; in this case let $C=\left\{x_{1} x_{2}\right\}$ for some fixed $x_{1} x_{2} \in E(S)$. Let $c=|C|$ and $\alpha^{*}=\min \left(\lceil\alpha|E|\rceil,\left\lfloor\frac{1}{2}|E|\right\rfloor\right)$. In particular, $2 \alpha^{*} \leq|E|$, and if $\lceil\alpha|E|\rceil>\alpha^{*}$, then $\lceil\alpha|E|\rceil=\alpha^{*}+1$ and $|E|$ is odd. Moreover, $|E-C|=2\left\lfloor\frac{1}{2}|E|\right\rfloor$.

If $c=0$, then $2 \alpha^{*}+c=2 \alpha^{*}=2\lceil\alpha|E|\rceil \geq\lceil 2 \alpha|E|\rceil$. If $c=1$, then $E$ is odd, $2 \alpha \leq 1$, and $2 \alpha^{*}+c=|E| \geq\lceil 2 \alpha|E|\rceil$. Thus $2 \alpha^{*}+c \geq\lceil 2 \alpha|E|\rceil$.

Let $a_{i}$ be the number of edges in $A_{i}=E\left(V_{i}\right) \cup E\left(V_{i}, S\right)$, and let $b$ be the number of edges in $E(S)-C$. Set $b_{1}=\max \left(\alpha^{*}-a_{1}, 0\right), b_{2}=b-b_{1}$, and let $E_{i}$ contain the edges of $A_{i}$ and $b_{i}$ edges of $E(S)-C$, such that $E_{1}$ and $E_{2}$ are disjoint. Then $\left|E_{1}\right|=a_{1}+b_{1} \geq \alpha^{*}$ and either $\left|E_{2}\right|=a_{2}+b_{2} \geq a_{2}+b \geq \alpha^{*}$ or $\left|E_{2}\right|=a_{2}+b_{2} \geq$ $a_{2}+b+a_{1}-\alpha^{*} \geq \alpha^{*}$. Therefore $\left|E_{i} \cup C\right| \geq\lceil\alpha|E|\rceil$ in all cases.

We construct a graph $\bar{G} \in \mathcal{H}_{G}$ as follows: insert trees on the leaves corresponding to vertices of $V_{1}$ and $V_{2}$ arbitrarily. If $C \neq \emptyset$, let $l_{1} l_{2}$ be the non-tree edge in $\bar{G}$ corresponding to the edge $x_{1} x_{2}$ in $G$. For $s \in S, s \notin\left\{x_{1}, x_{2}\right\}$, let $L_{s, i}$ be the set of leaves corresponding to $s$ that are endpoints of edges in $E_{i}$. Insert a tree on the leaves corresponding to $s$ such that a removal of an edge $e_{s}$ separates the leaves $L_{s, 1}$ from $L_{s, 2}$. For $F=\left\{e_{s} \mid s \in S\right\} \cup\left\{l_{1} l_{2}\right\}$, the graph $\bar{G}-F$ consists of two graphs $\bar{G}_{i}$ containing the set of leaves $L_{i}$ of size at least $\left|L_{i}\right| \geq 2 \alpha^{*}+c \geq\lceil\alpha|L|\rceil$. Therefore $F$ is an $\alpha$-edge bisection of $\bar{G} \in \mathcal{H}$ implying $\mathrm{bw}_{v}^{*}(G ; \alpha) \geq \operatorname{bw}_{e}(\bar{G}, L ; \alpha)$ and proving the first inequality.

We establish a lower bound on the minor crossing numbers of graphs using Lemma 7 together with the following theorem of Alexandrov and Djidjev [3]:

Theorem 8 ([3], Theorem 3.1) Let $G$ be an n-vertex graph with non-negative vertexweights $w$ such that $G$ is embeddable in an orientable surface of Euler genus $g$. For any $\varepsilon \in(0,1)$, there exists a set $S$, such that $|S| \leq 4 \sqrt{\left(\frac{g}{2}+\frac{1}{\varepsilon}\right) n}$ and no component of $V(G-S)$ has weight more than $\varepsilon w(G)$.

Theorem 8 or some similar result on separators together with the standard iterative technique for producing an $\alpha$-edge bisection of size at most $\frac{c \Delta(G)}{1-\varepsilon^{p}} n^{p}$ using separators of size at most $c n^{p}, 0<p<1,[11,18,29,30]$ implies the following:

Corollary 9 Let $G$ be an $n$-vertex graph of maximum degree $\Delta$ embeddable in an orientable surface of Euler genus $g$. Let $L \subset V$ and $\varepsilon \in(0,1), \alpha \in\left(0, \frac{1}{2}\right]$. Then there exists an $\alpha$-edge bisection of $G$ with respect to $L$ of size at most $\frac{4 \Delta}{1-\sqrt{\varepsilon}} \sqrt{\left(\frac{g}{2}+\frac{1}{\varepsilon}\right) n}$. 
Proof Assign weight 1 to all vertices from the set $L$ and 0 to all remaining vertices. According to Theorem 8 , there is a set $S_{1} \subseteq V(G)$ of size $4 \sqrt{\left(\frac{g}{2}+\frac{1}{\varepsilon}\right) n}$ whose removal leaves no component of weight larger than $\varepsilon|L|$. Let $C_{1}$ be the largest component; then we can group the other components into two sets $A_{1}, B_{1} \subseteq V(G)$, such that there are no edges between $A_{1}$ and $B_{1}$ in $G-S_{1}$, and $w\left(A_{1}\right), w\left(B_{1}\right) \leq(1-\alpha)|L|$. If $w\left(B_{1} \cup C_{1}\right) \leq(1-\alpha)|L|$, we are done, otherwise we proceed by separating $C_{1}$ using a set $S_{1}^{\prime}$ of size at most $4 \sqrt{\left(\frac{g}{2}+\frac{1}{\varepsilon}\right) \varepsilon n}$ into components of weight at most $\varepsilon^{2}|L|$. Then we can add all but at most one (call it $C_{2}$ ) of those components to sets $A_{1}$ and $B_{1}$ obtaining $A_{2} \supseteq A_{1}, B_{2} \supseteq B_{1}$, such that $w\left(A_{1}\right), w\left(B_{1}\right) \leq(1-\alpha)|L|$ and there are no edges between $A_{2}$ and $B_{2}$ in $G-S_{2}, S_{2}=S_{1} \cup S_{1}^{\prime}$ : the same argument as before applies. Iterating this procedure, we obtain sequences $A_{1} \subseteq \cdots \subseteq A_{k}, B_{1} \subseteq \cdots \subseteq B_{k}$, $C_{1} \supseteq \cdots \supseteq C_{k}$, and $S_{1} \subseteq \cdots \subseteq S_{k}$, such that $w\left(A_{i}\right) \leq w\left(B_{i}\right) \leq(1-\alpha)|L|, w\left(C_{i}\right) \leq$ $\varepsilon^{i}|L|$ for $i=1, \ldots, k-1, w\left(C_{k}\right)=0$, and

$$
\left|S_{k}\right| \leq \sum_{i=0}^{k} 4 \sqrt{\left(\frac{g}{2}+\frac{1}{\varepsilon}\right) \varepsilon^{i} n} \leq \frac{4}{1-\sqrt{\varepsilon}} \sqrt{\left(\frac{g}{2}+\frac{1}{\varepsilon}\right) n} .
$$

Let $F$ be the set of at most $\Delta\left|S_{k}\right|$ edges, incident with vertices of $S_{k}$. The vertices of $S_{k}$ are isolated vertices in $G-F$ and can be properly distributed among the sets $A_{k}$ and $B_{k}$, so that we obtain $A_{k+1}$ and $B_{k+1}$ that are a partition of $V(G)$ with $w\left(A_{k+1}\right), w\left(B_{k+1}\right) \geq\lfloor\alpha|L|\rfloor$. Thus $F$ is an $\alpha$-edge bisection of $G$ with respect to $L$.

Theorem 10 Let $G=(V, E)$ be a graph with minimum degree at least three, $\Sigma$ an orientable surface of Euler genus $g$. For every $\alpha \in\left(0, \frac{1}{2}\right]$, there exists $c_{g}>0$ such that

$$
\operatorname{mcr}(G, \Sigma) \geq c_{g} \mathrm{bw}_{v}^{*}(G ; \alpha)^{2}-4|E|
$$

Proof We prove the theorem with $c_{g, \varepsilon}=\frac{\varepsilon(1-\sqrt{\varepsilon})^{2}}{128(g \varepsilon+2)}$ for any $\varepsilon \in(0,1)$. Let $\bar{G}^{\prime}$ be a realizing graph of $G$, i.e., $\operatorname{mcr}(G, \Sigma)=\operatorname{cr}\left(\bar{G}^{\prime}, \Sigma\right)$. As $G$ has minimum degree three, we may assume that $\bar{G}^{\prime}$ is cubic (cf. [6]) and we can obtain a graph $\bar{G} \in \mathcal{H}_{G}$ by subdividing each original edge of $\bar{G}^{\prime}$ twice. Using a standard technique of Leighton [17], we will prove that

$$
\operatorname{cr}(\bar{G}, \Sigma) \geq c_{g, \varepsilon} \mathrm{bw}_{e}(\bar{G}, L ; \alpha)^{2}-4|E|+2|V| .
$$

Then Lemma 7 will imply $\operatorname{mcr}(G, \Sigma) \geq c_{g, \varepsilon}\left(\mathrm{bw}_{v}^{*}(G ; \alpha)-1\right)^{2}-4|E|+2|V| \geq$ $c_{g, \varepsilon} \mathrm{bw}_{v}^{*}(G ; \alpha)^{2}-4|E|$.

Let $D$ be an optimal drawing of $\bar{G}$ and replace every crossing of $D$ with a new vertex. We get graph $G_{D}$ on $\operatorname{cr}(\bar{G}, \Sigma)+|L|+|I|=\operatorname{cr}(\bar{G}, \Sigma)+4|E|-2|V|$ vertices, whose maximum degree is four, embedded in $\Sigma$. (Recall that the internal vertices $I$ and the leaf vertices $L$ were defined before Lemma 7.) Assign the weight 1 to every leaf vertex and weight zero to other vertices in $G_{D}$. Corollary 9 implies that there 
exists an $\alpha$-edge bisection $F_{D}$ of $G_{D}$ with respect to $L$ of size

$$
\left|F_{D}\right| \leq \frac{16}{1-\sqrt{\varepsilon}} \sqrt{\left(\frac{g}{2}+\frac{1}{\varepsilon}\right)\left|V_{D}\right|}
$$

Each edge of $F_{D}$ corresponds to a unique edge of $\bar{G}$, but two edges in $F_{D}$ may correspond to the same edge of $\bar{G}$. Thus the set $F$ of edges of $\bar{G}$ corresponding to the edges of $F_{D}$ has size at most $\left|F_{D}\right|$. Since $F$ is an $\alpha$-edge bisection of $\bar{G}$ with respect to $L$, we established

$$
\frac{16}{1-\sqrt{\varepsilon}} \sqrt{\left(\frac{g}{2}+\frac{1}{\varepsilon}\right)} \sqrt{\operatorname{cr}(\bar{G}, \Sigma)+4|E|-2|V|} \geq|F| \geq \mathrm{bw}_{e}(\bar{G}, L ; \alpha),
$$

which implies (3.1).

\section{The Embedding Method}

Let $H, G$ be two graphs. An embedding of $H$ into $G$ is a pair of injections $\omega=\langle\lambda, \Lambda\rangle$, $\lambda: V(H) \rightarrow V(G), \Lambda: E(H) \rightarrow\{P \mid P$ is a path in $G\}$, such that $\Lambda(e)$ is a path in $G$ from $\lambda(u)$ to $\lambda(v)$ for any edge $e=u v \in E(H)$. The paths $\Omega_{\omega}=\{\Lambda(e) \mid e \in$ $E(H)\}$ are called $\omega$-active paths. The edge congestion $\mu_{\omega}(e)$ of an edge $e \in E(G)$ is the number of active paths using $e$, and the vertex congestion $m_{\omega}(v)$ of a vertex $v \in V(G)$ is the number of active paths using the vertex $v \in V(G)$. Edge congestion $\mu_{\omega}$ and vertex congestion $m_{\omega}$ of the embedding $\omega$ are the maximum corresponding values over all the edges or vertices. Given an embedding of $H$ into $G$, the following theorem bounds the crossing number of $G$ in terms of the crossing number of $H$ :

Theorem 11 [27] Let $G$ be a graph of order $n, \omega$ an embedding of a graph $H$ into $G$ with edge congestion $\mu_{\omega}$ and vertex congestion $m_{\omega}$, and $\Sigma$ any surface. Then,

$$
\operatorname{cr}(G, \Sigma) \geq \frac{\operatorname{cr}(H, \Sigma)}{\mu_{\omega}^{2}}-\frac{n}{2}\left(\frac{m_{\omega}}{\mu_{\omega}}\right)^{2} .
$$

For our purposes, we need to refine the above statement. Let $\omega$ be an embedding of a graph $H$ into a graph $G$. For a pair of edges $e, f \in E(H)$ let $P$ be a component of $\Lambda(e) \cap \Lambda(f)$. Clearly, $P$ is a path in $G$. If $e$ and $f$ are adjacent and $P$ contains a $\lambda$-image of their common endvertex, then $P$ is a starting component, otherwise $P$ is a non-starting component of $\Lambda(e) \cap \Lambda(f)$. We denote with $o_{\omega}(e, f)$ the number of non-starting components of $\Lambda(e) \cap \Lambda(f)$. Let $o_{\omega}=\sum_{\{e, f\} \in \Pi_{\omega}} o_{\omega}(e, f)$. With $\Pi_{\omega} \subseteq\left(\begin{array}{c}E(H) \\ 2\end{array}\right)$ we denote the set of entangled edge pairs of $H$ : a pair $\{e, f\} \subseteq E(H)$ is entangled, if $o_{\omega}(e, f)>0$.

Theorem 12 Let $G$ be a graph, $\Sigma$ any surface, and $\omega=(\lambda, \Lambda)$ an embedding of a graph $H$ into $G$ with edge congestion $\mu_{\omega}$. Then $\operatorname{cr}(G) \geq \frac{1}{\mu_{\omega}^{2}}\left\{\operatorname{cr}(H, \Sigma)-o_{\omega}\right\}$. 
Proof Let $D$ be a drawing of $G$ and let $D^{\prime}$ be the subdrawing, induced by the edges of $\Lambda(E(H))$. Using the embedding $\omega$ as in [27], we construct a drawing $D_{H}$ of $H$ as follows. First, we draw each vertex $v \in V(H)$ into the $D$-image of $\lambda(v)$. Second, we draw each edge $e \in E(H)$ in a small neighborhood of the drawing $D[\Lambda(e)]$ of the embedding path $\Lambda(e)$ parallel with that path. We say that such $D_{H}$ respects $D$ and $\omega$.

In $D_{H}$, there are precisely two types of crossings. Crossings of type (i) arise in small neighborhood of some crossing $x$ of $D$ : if $\Lambda(e)$ and $\Lambda(f)$ each uses a different edge of $G$ that crosses at $x$, then $e$ and $f$ cross in a crossing of type (i) in the neighborhood of $x$.

Crossings of type (ii) arise in small neighborhoods of some vertex $v$ of $G$ : if $v \in$ $\Lambda(e) \cap \Lambda(f)$, then $e$ and $f$ may cross in a crossing of type (ii) in the neighborhood of $v$.

The construction of such a drawing $D_{H}$ alone implies Theorem 11, as there are at most $\mu_{\omega}^{2}$ crossings of type (i) at every crossing of $D$, and at most $m_{\omega}^{2} / 2$ crossings of type (ii) at every vertex of $G$. The improvement follows from elimination and a more sharp counting of crossings of type (ii).

The sharper counting relies on the obvious fact that crossings of type (ii) appear only at vertices of $G$. Thus, they can appear only at vertices of $\Lambda(e) \cap \Lambda(f)$ for some $e, f \in E(H)$.

We claim that there exists $D_{H}$ with (a) at most one $e, f$-crossing of type (ii) per non-starting component of $\Lambda(e) \cap \Lambda(f)$ and with no type (ii) $e, f$-crossings at starting components of $\Lambda(e) \cap \Lambda(f)$.

Assume no such $D_{H}$ exists and let $\bar{D}$ be the drawing respecting $D$ and $\omega$ with the smallest number of violations of (a). Further, assume that $P \subseteq G$ is a component of $\Lambda(e) \cap \Lambda(f)$.

If $v \in P$ is an endvertex of both $\Lambda(e)$ and $\Lambda(f)$ and $e, f$ cross in $\bar{D}$ in a small neighborhood of $w \in P$, we can flip $e$ and $f$ in a small neighborhood of $P$ such that the crossing is eliminated. The new drawing still respects $D$ and $\omega$ as $P$ is a component of $\Lambda(e) \cap \Lambda(f)$, and both drawings $\bar{D}[e]$ and $\bar{D}[f]$ are routed in small neighborhood of $P$. But the new drawing has a smaller number of violations of (a) than $\bar{D}$, a contradiction to the choice of $\bar{D}$.

So we may assume that $P$ is a non-starting component with at least two type (ii) crossings of $e$ and $f$. Let $x, y$ be the vertices of $P$ in whose small neighborhoods in $\bar{D}$ the two crossings appear. If we flip the edges $e$ and $f$ in small neighborhoods of the crossings, the new drawing still respects $\bar{D}$ and $\omega$ as in the previous paragraph and has a smaller number of violations of (a) than $\bar{D}$, another contradiction to the choice of $\bar{D}$. We conclude that (a) holds.

In $D_{H}$, there are at most $\mu_{\omega}^{2}$ type (i) crossings at any crossing of $G$, and altogether at most $o_{\omega}$ type (ii) crossings. Thus,

$$
\mu_{\omega}^{2} \operatorname{cr}(G, \Sigma)+o_{\omega} \geq \operatorname{cr}(H, \Sigma),
$$

and the claim follows.

In special circumstances that are of interest in [5], Theorem 12 could be improved:

Theorem 13 Let $G$ be a graph and $\omega=(\lambda, \Lambda)$ an embedding of a graph $H$ into $G$ with edge congestion $\mu_{\omega}=1$, such that all $\omega$-entangled pairs of edges of $H$ have the 
same common endvertex $v$. Then any drawing $D$ of $G$ has at least $\operatorname{cr}(H, \Sigma)$ crossings in $D\left[\Omega_{\omega}\right]$ that do not involve two edges of $\Lambda$-images of $\omega$-entangled pairs of edges.

Proof Let $D_{H}$ be a drawing of $H$ respecting $D$ and $\omega$ with smallest number of type (ii) crossings, as in the proof of Theorem 12. We augment $D_{H}$ to $D_{H}^{\prime}$ and produce an embedding $\omega^{\prime}=\left(\lambda^{\prime}, \Lambda^{\prime}\right)$, such that $D_{H}^{\prime}$ respects $D$ and $\omega^{\prime}, \mu_{\bar{\omega}}=1, \Lambda^{\prime}(E(H))$ contain the same edges as $\Lambda(E(H))$, and $D_{H}^{\prime}$ has no type (ii) crossings.

If $D_{H}$ has no type (ii) crossings, then $\omega^{\prime}=\omega$ and $D_{H}^{\prime}=D_{H}$, otherwise let $x$ be a type (ii) crossing in a small $D_{H}$-neighborhood of a vertex $w \in V(G)$ and let $e, f$ be the two edges of $H$ crossing at $x$.

As $\mu_{\omega}=1, x$ is a non-starting component of $\Lambda(e) \cap \Lambda(f)$. So let $P_{e}$ and $P_{f}$ be the maximum common $w-v$ segments of $\Lambda(e)$ and $\Lambda(f)$, respectively. We alter $\omega=(\lambda, \Lambda)$ to $\omega^{\prime}=\left(\lambda, \Lambda^{\prime}\right)$, so that $\Lambda^{\prime}(e)$ uses $P_{f}$ and $\Lambda^{\prime}(f)$ uses $P_{e}$, but otherwise they are equal. By flipping $e$ and $f$ in a small $D_{H}$-neighborhood of $x$, we obtain $D_{H}^{\prime}$ that respects $\omega^{\prime}$. After performing such a change at every type (ii) crossing of $D_{H}$, the final $D_{H}^{\prime}$ respects the final $\omega^{\prime}$, and $D_{H}$ has no type (ii) crossings.

We further produce a drawing $D_{H}^{\prime \prime}$, which has no type (i) crossings on the $\Lambda$ images of the $\omega$-entangled pairs of $H$-edges. As all such edges are incident with $v$, we can uncross them at any type (i) crossing involving such edges, even if the two edges are not from the same entangled pair. The new drawing is still a drawing of $H$ and has at least $\operatorname{cr}(H, \Sigma)$ crossings, and we deduce that all these crossings are type (i) crossings appearing in small neighborhoods of crossings of $D$. As $\mu_{\omega}=1$, each such type (i) crossing corresponds to a unique crossing of $D$, so $D$ has at least $\operatorname{cr}(H, \Sigma)$ crossings of which none involves two $\Lambda$-images of $\omega$-entangled pairs of edges.

The ideas behind Theorem 13 could be applied in more general settings, too. By eliminating the crossings in a specific setting of a given embedding and a given drawing respecting that embedding, the bounds could be further improved, either by decreasing the multiplicative factor (in our case $\mu_{\omega}$ ) or by decreasing the subtracted constant (in our case $o_{\omega}$ ).

Let $G$ be a graph and $\bar{G}$ a realizing graph of $G$ in some surface $\Sigma$. For a vertex $v$ in $V(G)$, let $T_{v}$ be the tree in $\bar{G}$ that is contracted to $v$. For any path $P=u_{0} \cdots u_{t}$ in $G$ of positive length, we define its lift $\bar{P}$ to be the path in $\bar{G}$ that uses every edge $e_{i}$ of $\bar{G}$ corresponding to $u_{i-1} u_{i} \in E(G), i=1, \ldots, t$, and connects the edges $e_{i}$ and $e_{i+1}$ with the unique path in $T_{u_{i}}$ connecting their endvertices. Formally, $\bar{P}=$ $e_{1} T_{u_{1}} e_{2} T_{u_{2}} \cdots T_{u_{t-1}} e_{t}$.

If $\omega=\langle\lambda, \Lambda\rangle$ is an embedding of a graph $H$ into $G$ and $\bar{G}$ a realizing graph of $G$ in some surface $\Sigma$, then a lift of $\omega$ is any embedding $\bar{\omega}=\langle\bar{\lambda}, \bar{\Lambda}\rangle$ of $H$ into $\bar{G}$, for which $\bar{\lambda}(v) \in V\left(T_{\lambda(v)}\right)$ for every $v \in V(H)$; and, for $e=u v, \bar{\Lambda}(e)$ is the path containing the lift $\bar{P}$ of $\Lambda(e)$ extended by the path connecting $\bar{\lambda}(u)$ with the initial vertex of $\bar{P}$ and the path connecting the endvertex of $\bar{P}$ with $\bar{\lambda}(v)$. Note that we have the freedom of choosing $\bar{\lambda}(v) \in T_{\lambda(v)}$. After that, the lifts of paths are uniquely defined.

Lemma 14 Let $G, H$ be two graphs and $\bar{G}$ a realizing graph of $G$ in some surface $\Sigma$. Further, let $\omega=\langle\lambda, \Lambda\rangle$ be an embedding of $H$ into $G$ and let $\bar{\omega}=\langle\bar{\lambda}, \bar{\Lambda}\rangle$ be a lift of $\omega$. Then $o_{\bar{\omega}}(e, f)=o_{\omega}(e, f)$ for any pair of edges $e, f \in E(H)$ and consequently $o_{\bar{\omega}}=o_{\omega}$. 
Proof Let $e, f \in E(H)$ and let $P=v_{1} e_{1} v_{2} e_{2} \cdots e_{k-1} v_{k}$ be a component of $\Lambda(e) \cap \Lambda(f)$. If $P$ is a starting component, then (by symmetry) we may assume $v_{1}$ is the $\lambda$-image of a common endvertex $w$ of $e$ and $f$. By definition of the lift $\bar{\omega}$, $\bar{\lambda}(w)$ is a vertex in $T_{v_{1}}$. Furthermore, $\bar{\Lambda}(e)$ and $\bar{\Lambda}(f)$ contain the edges $e_{1}, \ldots, e_{k}$, and as there are unique endvertices of corresponding edges in $T_{v_{i}}, i=1, \ldots, k-1$ and unique paths connecting these endvertices in $T_{v_{i}}$, the respective lifts of $P$ into $\bar{G}$ are in the same component $\bar{P}$ of $\bar{\Lambda}(e) \cap \bar{\Lambda}(f)$ in $\bar{G}$. In $T_{v_{k}}$, however, only the endvertex $w^{\prime}$ of $e_{k-1}$ is a common $T_{v-k}$-leaf of the lift, the other leaf is distinct for each of $\bar{\Lambda}(e), \bar{\Lambda}(f)$. But, as $T_{v_{k}}$ contains no cycles, $\bar{\Lambda}(e) \cap \bar{\Lambda}(f) \cap T_{v_{k}}$ has only one component that is a path $\bar{P}^{\prime}$ and contains $w^{\prime}$. As $w^{\prime}$ is in $\bar{P}$ and $\bar{P}^{\prime}, \bar{P}^{\prime}$ is a part of $\bar{P}$. Thus there is a unique component of $\bar{\Lambda}(e) \cap \bar{\Lambda}(f) \cap T_{v_{k}}$ that corresponds to $P$. A similar reasoning applies if $P$ is not a starting component: in that case, the $T_{v_{1}}$ endvertex of $e_{1}$ defines the only component $\bar{P}$ of $\bar{\Lambda}(e) \cap \bar{\Lambda}(f) \cap T_{v_{1}}$ which contains $e_{1} T_{u_{1}} e_{2} T_{u_{2}} \cdots T_{u_{t-1}} e_{t}$. Thus, for every component $P$ of $\Lambda(e) \cap \Lambda(f)$ there exists a component $\bar{P}$ of $\bar{\Lambda}(e) \cap \bar{\Lambda}(f)$. As $\bar{\Lambda}(e)$ is a path for every $e \in E(H)$, there is only one component $\bar{P}$ for each $P$, and the claim follows.

We define $s_{\omega}(v)$ to be the number of $\omega$-active paths starting at $v \in V(G)$ and $t_{\omega}(v)$ to be the number of active paths passing through $v$. Then $m_{\omega}(v)=s_{\omega}(v)+t_{\omega}(v)$, but we define $v_{\omega}(v)=\left\lfloor\frac{1}{2} s_{\omega}(v)\right\rfloor+t_{\omega}(v)$ and $v_{\omega}=\max \left(\max _{v \in V(G)} v_{\omega}(v)\right.$, $\left.\max _{e \in E(G)} \mu_{\omega}(e)\right)$. As follows, this refinement strengthens the translation of the embedding method to the minor crossing number in such a way that the result generalizes the lower bound on $\operatorname{mcr}(G, \Sigma)$ in terms of $\operatorname{cr}(G, \Sigma)$ and $\Delta(G)$ from $[6,10]$.

Theorem 15 Let $G$ be a graph and $\omega=\langle\lambda, \Lambda\rangle$ an embedding of a connected nonempty graph $H$ into $G$. Then,

$$
\operatorname{mcr}(G, \Sigma) \geq \frac{1}{v_{\omega}^{2}}\left\{\operatorname{cr}(H, \Sigma)-o_{\omega}\right\}
$$

Proof Let $\bar{G}$ be a realizing graph of $G$.

Claim 1: There exists a lift $\bar{\omega}: H \rightarrow \bar{G}, \bar{\omega}=\langle\bar{\lambda}, \bar{\Lambda}\rangle$, such that for every $v \in V(G)$ and $e \in E\left(T_{v}\right), \mu_{\bar{\omega}}(e) \leq\left\lfloor\frac{1}{2} s_{\omega}(v)\right\rfloor+t_{\omega}(v)$.

Claim 1 implies $\mu_{\bar{\omega}} \leq v_{\omega}$. As $o_{\bar{\omega}}=o_{\omega}$ by Lemma 14, Theorem 12 applied to $\bar{G}$ implies the theorem.

Now we prove Claim 1. According to a previous remark, it is enough to define $\bar{\lambda}\left(v^{\prime}\right)$ for any $v^{\prime} \in V(H)$ so that the bound of Claim 1 holds. Let $v$ be a vertex of $G$ and $T_{v}$ the tree in $\bar{G}$ contracted to $v$. If $s_{\omega}=0$, then there are $t_{\omega}$ active paths using $v$, none of them as a starting vertex. In $\bar{\omega}$, at most the corresponding $t_{\omega}$ lifted paths can use any $e \in T_{v}$, so the claim holds. Therefore we may assume that $s_{\omega} \geq 1$, so $v=\lambda\left(v^{\prime}\right)$ for some $v^{\prime} \in V(H)$ with $d_{v^{\prime}} \geq 1$.

As the paths going through $v$ contribute at most $t_{\omega}(v)$ to $\mu_{\bar{\omega}}(e)$ as in the previous paragraph, we may for simplicity assume that $t_{\omega}(v)=0$. Let $e=u_{1} u_{2}$ be an edge of $T_{v}$. The forest $T_{v}-e$ has two components $T_{1}$ and $T_{2}$ with $u_{i} \in V\left(T_{i}\right), i=1,2$. Let 
$E_{i}$ be the set of all original edges of $G$, incident with $T_{i}$, and let $\mu_{i}=\sum_{e \in E_{i}} \mu_{\omega}(e)$. Note that, $(*)$ if $\bar{\lambda}\left(v^{\prime}\right) \in V\left(T_{i}\right)$, then $\mu_{\bar{\omega}}(e)=\mu_{3-i}$. If $\mu_{i}<\mu_{3-i}$, we direct the edge $e$ from $u_{i}$ to $u_{3-i}$, otherwise we leave the edge $e$ undirected. With $T_{e}$ we denote the component $T_{3-i}$ and with $T_{e}^{\prime}$ the component $T_{i}$. If $\mu_{1}=\mu_{2}$, then $T_{e, u_{i}}$ denotes the tree $T_{i}$. With $\mu(T)$ we denote the sum of $\mu_{\omega}(e)$ for all original edges incident with $T$.

Claim 2: Each vertex $u \in V\left(T_{v}\right)$ has at most one incident outgoing edge. Suppose the edges $e$ and $f$ are both directed away from $u$. Since $T_{e}^{\prime}$ contains $T_{f}$ and $T_{f}^{\prime}$ contains $T_{e}$, this would imply $\mu\left(T_{f}\right) \leq \mu\left(T_{e}^{\prime}\right)<\mu\left(T_{e}\right) \leq \mu\left(T_{f}^{\prime}\right)$, a contradiction.

Claim 3: If $u \in V\left(T_{v}\right)$ is incident with an undirected edge, then there is no outgoing edge incident with $u$. Let $u$ be incident with an outgoing edge $e$ and an undirected edge $f=u u^{\prime}$. Since $f$ is undirected, $\mu\left(T_{f, u}\right)=\mu\left(T_{f, u^{\prime}}\right)=\frac{1}{2} m_{v}$. Then, $\mu\left(T_{e}\right)>$ $\mu\left(T_{e}^{\prime}\right) \geq \mu\left(T_{f, u^{\prime}}\right)=\frac{1}{2} m_{v}$, since $T_{f, u^{\prime}}$ is a subtree of $T_{e}$. This contradicts $\mu\left(T_{e}\right)+$ $\mu\left(T_{e}^{\prime}\right)=m_{v}$.

Claim 4: $u \in V\left(T_{v}\right)$ can have at most two incident undirected edges. Let $e_{i}=u u_{i}$, $i=1,2,3$, be three incident undirected edges. Then $\mu_{1}=\mu_{2}+\mu_{3}+\mu^{\prime}, \mu_{2}=\mu_{1}+$ $\mu_{3}+\mu^{\prime}$, and $\mu_{3}=\mu_{1}+\mu_{2}+\mu^{\prime}$ for $\mu_{i}=\mu\left(T_{e_{i}, u_{i}}\right)$. Since $\mu_{i}, \mu^{\prime} \geq 0$, this implies $\mu_{1}=\mu_{2}=\mu_{3}=\mu^{\prime}=0$, contradicting $d_{v^{\prime}} \geq 1$.

Claim 5: The subgraph of $T_{v}$ induced by undirected edges is connected. By Claim 2 and Claim 3, the unique path $e_{1} \cdots e_{t}$ of $T_{v}$ connecting two undirected edges $e_{1}$ and $e_{t}$ has only incoming edges, therefore the path can contain undirected edges only.

Claims 4 and 5 establish that the subgraph spanned by the undirected edges of $T_{v}$ is a connected graph $P$ of maximum degree two, and by Claim 2 and 4 , all edges of $T$ incident with $P$ are directed into $P$. If there is no undirected edge, then $T_{v}$ is a directed acyclic graph and must have a vertex $P=u$ of out-degree zero. If we embed $v^{\prime}$ into $P$, then Claim 1 follows by $(*)$.

The inequality $\operatorname{mcr}(G, \Sigma) \geq \operatorname{cr}(G, \Sigma) /\left\lfloor\frac{\Delta(G)}{2}\right\rfloor^{2}$, proved in [10] for $\Delta(G)=4$ and in [6] for general $\Delta(G)$, is a simple consequence of Theorem 15: If we embed $G$ into $G$ using the canonical injection $\iota$, then $s_{\iota}=\Delta(G)$ and $t_{\iota}=0$ imply $v_{\iota}=\left\lfloor\frac{\Delta(G)}{2}\right\rfloor$, which together with $o_{\iota}=0$ implies the inequality.

Note that there are two ways of using an embedding $\omega: H \rightarrow G$ to obtain a lower bound for $\operatorname{mcr}(G, \Sigma)$ in terms of $\operatorname{cr}(H, \Sigma)$. We can apply it directly using Theorem 15 , in which case the lower bound is roughly $\operatorname{cr}(H, \Sigma) / v_{\omega}^{2}$, or we can first apply Theorem 12 to obtain a lower bound on $\operatorname{cr}(G, \Sigma)$, and then use the embedding $\iota: G \rightarrow G$ from the previous paragraph, in which case we obtain a bound, roughly equal to $4 \operatorname{cr}(H, \Sigma) /\left(\Delta \mu_{\omega}\right)^{2}$. The direct approach is preferable whenever $2 v_{\omega} \leq \Delta \mu_{\omega}$, otherwise the indirect approach yields a better bound.

\section{Applications}

\subsection{Hypercubes}

In [6], Bokal, Fijavž and Mohar have shown that for the $n$-dimensional hypercube $Q_{n}$, if $n \geq 4$, we have

$$
\frac{4^{n}}{5 n^{2}}(1+o(1)) \leq \operatorname{mcr}\left(Q_{n}\right) \leq \frac{4^{n}}{8}(1+o(1)) .
$$


We improve on these bounds.

Theorem 16 For the $n$-dimensional hypercube $Q_{n}$, bw $_{v}\left(Q_{n}\right) \geq\left(\begin{array}{c}n \\ \left\lfloor\frac{n}{2}\right\rfloor-1\end{array}\right)=\frac{2^{n+1}}{\sqrt{2 \pi n}}(1-$ $o(1))$.

Proof We prove the claim for odd $n$. The even case is similar. Consider an optimal vertex bisection $S$ of edges of $Q_{n}$, which separates the hypercube into $G_{1}=\left(V_{1}, E_{1}\right)$ and $G_{2}=\left(V_{2}, E_{2}\right),\left|V_{1}\right| \geq\left|V_{2}\right|$, such that $\left|E_{1}\right| \leq 2^{n-2} n$ and $\left|E_{2}\right| \leq 2^{n-2} n$. By contradiction, we prove

$$
|S| \geq\left(\begin{array}{c}
n \\
\left\lfloor\frac{n}{2}\right\rfloor-1
\end{array}\right)=\frac{2^{n+1}}{\sqrt{2 \pi n}}(1-o(1)) .
$$

For $A \subseteq V$, define the vertex boundary of $A$ as $\partial_{v}(A)=\{u \in V-A$ : there exists $w \in A, u w \in E\}$ and the edge boundary of $A$ as $\partial_{e}(A)=\{u v \in E: u \in A, v \in V-A\}$.

Case 1: $\left|V_{1}\right| \leq 2^{n-1}+\left(\begin{array}{c}n \\ \left\lfloor\frac{n}{2}\right\rfloor\end{array}\right)$. Since $\left|V_{1}\right| \geq\left|V_{2}\right|$, this implies

$$
\begin{aligned}
\left|V_{2}\right| & =2^{n}-\left|V_{1}\right|-|S|>2^{n-1}-\left(\begin{array}{c}
n \\
\left\lfloor\frac{n}{2}\right\rfloor
\end{array}\right)-\left(\begin{array}{c}
n \\
\left\lfloor\frac{n}{2}\right\rfloor-1
\end{array}\right) \\
& =\left(\begin{array}{l}
n \\
0
\end{array}\right)+\left(\begin{array}{l}
n \\
1
\end{array}\right)+\cdots+\left(\begin{array}{c}
n \\
\left\lfloor\frac{n}{2}\right\rfloor-2
\end{array}\right), \\
\left|V_{2}\right| & \leq 2^{n-1}=\left(\begin{array}{l}
n \\
0
\end{array}\right)+\left(\begin{array}{l}
n \\
1
\end{array}\right)+\cdots+\left(\begin{array}{c}
n \\
\left\lfloor\frac{n}{2}\right\rfloor-1
\end{array}\right)+\left(\begin{array}{c}
n \\
\left\lfloor\frac{n}{2}\right\rfloor
\end{array}\right) .
\end{aligned}
$$

Distinguish two subcases. If

$$
\left|V_{2}\right| \leq\left(\begin{array}{l}
n \\
0
\end{array}\right)+\left(\begin{array}{l}
n \\
1
\end{array}\right)+\cdots+\left(\begin{array}{c}
n \\
\left\lfloor\frac{n}{2}\right\rfloor-1
\end{array}\right),
$$

then

$$
\left|V_{2}\right|=\left(\begin{array}{l}
n \\
0
\end{array}\right)+\left(\begin{array}{l}
n \\
1
\end{array}\right)+\cdots+\left(\begin{array}{c}
n \\
\left\lfloor\frac{n}{2}\right\rfloor-2
\end{array}\right)+\alpha\left(\begin{array}{c}
n \\
\left\lfloor\frac{n}{2}\right\rfloor-1
\end{array}\right),
$$

for some $0<\alpha \leq 1$. According to Bollobás and Leader [8, Corollary 2],

$$
\left|\partial_{v}\left(V_{2}\right)\right| \geq(1-\alpha)\left(\begin{array}{c}
n \\
\left\lfloor\frac{n}{2}\right\rfloor-1
\end{array}\right)+\alpha\left(\begin{array}{c}
n \\
\left\lfloor\frac{n}{2}\right\rfloor
\end{array}\right)>\left(\begin{array}{c}
n \\
\left\lfloor\frac{n}{2}\right\rfloor-1
\end{array}\right) .
$$

This, however, contradicts

$$
\left|\partial_{v}\left(V_{2}\right)\right| \leq|S|<\left(\begin{array}{c}
n \\
\left\lfloor\frac{n}{2}\right\rfloor-1
\end{array}\right) .
$$

If

$$
\left|V_{2}\right|>\left(\begin{array}{l}
n \\
0
\end{array}\right)+\left(\begin{array}{l}
n \\
1
\end{array}\right)+\cdots+\left(\begin{array}{c}
n \\
\left\lfloor\frac{n}{2}\right\rfloor-1
\end{array}\right)
$$


then

$$
\left|V_{2}\right|=\left(\begin{array}{l}
n \\
0
\end{array}\right)+\left(\begin{array}{l}
n \\
1
\end{array}\right)+\cdots+\left(\begin{array}{c}
n \\
\left\lfloor\frac{n}{2}\right\rfloor-1
\end{array}\right)+\alpha\left(\begin{array}{c}
n \\
\left\lfloor\frac{n}{2}\right\rfloor
\end{array}\right)
$$

Similarly,

$$
\left|\partial_{v}\left(V_{2}\right)\right| \geq(1-\alpha)\left(\begin{array}{c}
n \\
\left\lfloor\frac{n}{2}\right\rfloor
\end{array}\right)+\alpha\left(\begin{array}{c}
n \\
\left\lfloor\frac{n}{2}\right\rfloor+1
\end{array}\right)=\left(\begin{array}{c}
n \\
\left\lfloor\frac{n}{2}\right\rfloor
\end{array}\right),
$$

a contradiction again.

Case 2: $\left|V_{1}\right|>2^{n-1}+\left(\begin{array}{c}n \\ \left\lfloor\frac{n}{2}\right\rfloor\end{array}\right)$. We have $2\left|E_{1}\right|+\left|\partial_{e}\left(V_{1}\right)\right|=\left|V_{1}\right| n$, implying

$$
\begin{gathered}
2^{n-1} n+\left|\partial_{e}\left(V_{1}\right)\right|>2^{n-1} n+\left(\begin{array}{c}
n \\
\left\lfloor\frac{n}{2}\right\rfloor
\end{array}\right) n, \\
\left|\partial_{e}\left(V_{1}\right)\right|>\left(\begin{array}{c}
n \\
\left\lfloor\frac{n}{2}\right\rfloor
\end{array}\right) n .
\end{gathered}
$$

This is a contradiction to

$$
\left|\partial_{e}\left(V_{1}\right)\right| \leq\left|\partial_{e}(S)\right| \leq|S| n<\left(\begin{array}{c}
n \\
\left\lfloor\frac{n}{2}\right\rfloor-1
\end{array}\right) n .
$$

Applying Theorem 16 in combination with Lemma 7 and Theorem 10, we obtain the following corollary:

Corollary 17 For every orientable surface $\Sigma$ of Euler genus $g$, there exists a constant $c_{g}>0$, such that

$$
\operatorname{mcr}\left(Q_{n}, \Sigma\right) \geq c_{g} \frac{4^{n}}{n}(1-o(1))
$$

for the n-dimensional hypercube $Q_{n}$.

For the sake of completeness, we provide an upper bound:

Theorem 18 For the n-dimensional hypercube $Q_{n}$,

$$
\operatorname{mcr}\left(Q_{n}\right) \leq \frac{4^{n}}{\sqrt{\pi n}}-2 n
$$

Proof We make a staircase drawing of $Q_{n}$ by first identifying the vertices of $Q_{n}$ with the subsets of $[n]$. Each vertex corresponds to a line in the drawing; lines whose sets have even number of elements are horizontal, lines whose sets have odd number of elements are vertical. Lines intersect other lines only when the cardinalities of the two sets differ by 1 . This gives the bound

$$
\operatorname{mcr}\left(Q_{n}\right) \leq \sum_{i=1}^{n-2}\left(\begin{array}{l}
n \\
i
\end{array}\right)\left(\begin{array}{c}
n \\
i+1
\end{array}\right)=\frac{n 4^{n} \Gamma\left(n+\frac{1}{2}\right)}{\sqrt{\pi} \Gamma(n+2)}-2 n \leq \frac{4^{n}}{\sqrt{\pi n}}-2 n
$$


A similar approach can be applied to the Hamming graphs $K_{p}^{n}=\prod_{i=1}^{n} K_{p}$. They are $n(p-1)$-regular, have $p^{n}$ vertices, $n(p-1) p^{n} / 2$ edges, and are a natural generalization of hypercubes, which are Hamming graphs for $p=2$. It is known that $\operatorname{cr}\left(K_{p}^{n}\right)=\Theta\left(p^{2 n+2}\right)$ [32], thus the embedding method lower bound gives $\operatorname{mcr}\left(K_{p}^{n}\right)=$ $\Omega\left(\frac{p^{2 n}}{n^{2}}\right)$. Combining Theorem 10 with the vertex boundary estimations in [12] and the approach used in Theorem 16, we get a better estimate $\operatorname{mcr}\left(K_{p}^{n}\right)=\Omega\left(\frac{p^{2 n}}{n}\right)$.

\subsection{String Representation of Graphs}

A graph is called a string graph, if its vertices are represented by simple curves in the plane, and two vertices are connected by an edge if and only if the corresponding simple curves intersect. Benzer [4] was motivated by biology and Sinden [28] by electrical engineering to ask which graphs are string graphs. Ron Graham deserves much credit for recognizing the importance of the problem and making it known. Although Kratochvíl $[14,15]$ showed that the recognition problem of string graphs is NP-hard, only recently were Pach and Tóth [20] and independently Schaefer, Sedgwick, and Štefankovič $[25,26]$ able to show that the recognition problem of string graphs is decidable and is in NP. The basis of this result is an upper bound on how many crossings a drawing proving that an $n$-vertex graph is a string graph may need (note that a pair of crossing curves may intersect many times, and this may be even needed for the string graph representation). We should also mention that Kratochvíl and Matoušek established a relationship between the minimum number of crossings in sting representations, weak representations, and strong representations in [16].

In view of this history, it is surprising that the following analogue of graph drawings has not been considered before. Represent the vertices of the graph $G$ by simple curves on the surface $\Sigma$, and make sure that any two curves representing the endpoints of an edge of $G$ intersect, but allow for intersection of curves representing non-adjacent vertices. Minimize the total number of intersections over all pairs of curves. We call this quantity minus $|E(G)|$ the string crossing number of $G$ on $\Sigma$, and denote it by $\operatorname{scr}(G, \Sigma)$. As before, we use $\operatorname{scr}(G)$ for $\operatorname{scr}\left(G, S_{0}\right)$. Note that $\operatorname{scr}($.) is analogous to $i$ (.) in Richter and Thomassen [24] and Juarez and Salazar [13], where it was applied to closed curves. We have an interesting observation, supported by Propositions 19 and 20: $\operatorname{scr}(G, \Sigma)$ is intimately related to $\operatorname{mcr}(G, \Sigma)$.

Proposition 19 Let $G$ be a graph. Then, $\operatorname{scr}(G, \Sigma) \leq 4 \operatorname{mcr}(G, \Sigma)$.

Proof Let $\bar{G}$ be a realizing graph of $G$ as in Lemma $2, T_{v}$ be the tree in $\bar{G}$ corresponding to $v \in V(G)$, and let $e_{1}, e_{2}, \ldots, e_{m}$ be the edges leaving $T_{v}$ in $\bar{G}$. Recall that all crossings in the optimal drawing of $\bar{G}$ occur between tree edges of different trees. Extend $T_{v}$ into a bigger tree by adding to it "half" of the edges $e_{1}, e_{2}, \ldots, e_{m}$ (till their midpoint). Draw now a closed curve $C_{v}^{\prime}$ "very near" around this extended tree in such a way that if $T_{u}$ and $T_{v}$ share an extended edge $e$ that $C_{u}^{\prime}$ and $C_{v}^{\prime}$ touch at the midpoint of $e$, but have no more points in common. To obtain the string $C_{v}$ cut open the closed curve $C_{v}^{\prime}$. Note that for $u v \in E(G),\left|C_{u} \cap C_{v}\right|=1$, and a crossing of $e \in E\left(T_{v}\right)$ and $f \in E\left(T_{u}\right)$ results in at most 4 common points of $C_{u}$ and $C_{v} . v \mapsto C_{v}$ is the required string representation of $G$. 
Proposition 20 For any graph $G$ with $t(G)$ tree components, $\operatorname{mcr}(G, \Sigma) \leq$ $\operatorname{scr}(G, \Sigma)+|E(G)|-|V(G)|+t(G)$.

Proof First we prove $\operatorname{mcr}(G, \Sigma) \leq \operatorname{scr}(G, \Sigma)+|E(G)|-|V(G)|$ for a graph $G$ with $\delta(G) \geq 2$. Assume that $v \mapsto C_{v}$ is a string representation of $G$ with a drawing $D$ that realizes $\operatorname{scr}(G, \Sigma)$. In other words, the strings intersect $|E(G)|+\operatorname{scr}(G, \Sigma)$ times in $D$. We can assume without loss of generality that no three curves pass through any point. Observe the $|E(G)|$ intersection points in the string representation that represent edges of $G$. If $C_{u}$ and $C_{v}$ have a point $p$ in common that represents an edge $e$ of $G$, choose vertices $u_{e} \in C_{u}$ and $v_{e} \in C_{v}$ very close to $p$ such that they can be connected with a curve $C_{e}$ not creating any additional crossings with $C_{u}, C_{v}$, or any additional $C_{f}$. Furthermore, we can make sure that the first and last point thus added to any curve $C_{v}$ is within the segment of $C_{v}$ bounded by the first and last crossings on $C_{v}$. We create a new graph $\hat{G}$ with a drawing $\hat{D}$ as follows: the points of this graph are the points $v_{e}$ on the curves $C_{v}$. The edges of $\hat{G}$ are drawn as follows: along the curve $C_{v}$ connecting neighboring points $v_{e}$ and $v_{f}$, and the curves $C_{e}$. We obtained a drawing $\hat{D}$ of a graph $\hat{G}$ containing $G$ as a minor. Since $\delta(G) \geq 2$, this drawing removes a crossing from both ends of each string; so it removes a total of $|V(G)|$ crossings from $D$. The number of crossings in $\bar{D}$ is therefore at most $\operatorname{scr}(G, \Sigma)+|E(G)|-|V(G)|$; wherever the curves $C_{u}$ and $C_{v}$ touch (not cross), we could eliminate an additional crossing.

To conclude, let $G$ be a general graph and let $G_{1}$ be the graph obtained from $G$ by removing all tree components of $G$. Clearly $\operatorname{mcr}\left(G_{1}, \Sigma\right)=\operatorname{mcr}(G, \Sigma)$, $\operatorname{scr}\left(G_{1}, \Sigma\right)=\operatorname{scr}(G, \Sigma),\left|E\left(G_{1}\right)\right|-\left|V\left(G_{1}\right)\right|=|E(G)|-|V(G)|+t(G)$, and every component in $G_{1}$ has at least one cycle. Create $G_{2}$ from $G_{1}$ by iteratively removing degree one vertices. Since every component of $G_{1}$ has a cycle, $G_{2}$ is nonempty, has the same number of components as $G_{1}, \delta\left(G_{2}\right) \geq 2, \operatorname{mcr}\left(G_{2}, \Sigma\right)=\operatorname{mcr}\left(G_{1}, \Sigma\right)$, $\operatorname{scr}\left(G_{2}, \Sigma\right)=\operatorname{scr}\left(G_{1}, \Sigma\right)$, and $\left|E\left(G_{2}\right)\right|-\left|V\left(G_{2}\right)\right|=\left|E\left(G_{1}\right)\right|-\left|V\left(G_{1}\right)\right|$. The lower bound follows by the previous lower bound applied to $G_{2}$.

Combining these propositions with known results on the minor crossing number from previous sections and from [6,7], we obtain bounds on the string crossing number for several families of graphs as well as general bounds. Perhaps most interesting is the following observation that follows from Proposition 19 and the results of [7], which claim that $\operatorname{mcr}(G) \leq c_{H}|V(G)|$ for $H$-minor-free graphs $G$ :

Corollary 21 For every graph $H$, there exists a constant $c_{H}$, such that every $H$ minor-free graph $G$ has string crossing number $\operatorname{scr}(G) \leq c_{H}|V(G)|$.

Extending Corollary 21 for general surfaces would require the extending the result of the $\operatorname{mcr}(G) \leq c_{H}|V(G)|$ to general surfaces for $H$-minor-free graphs $G$. The main tool of [7] for proving this result on the plane is planar decomposition of graphs. Thus, we would need to study such decompositions on general surfaces for such an extension.

In other words, Corollary 21 implies that graphs with no prescribed minor have their deficiency to being string graphs linear in their order. Proposition 20 with Corol- 
lary 17 implies that

$$
\operatorname{scr}\left(Q_{n}\right)=\Omega\left(\frac{4^{n}}{n}\right)=\Omega\left(\frac{\left|V\left(Q_{n}\right)\right|^{2}}{n}\right),
$$

thus exclusion of a minor is necessary in Corollary 21.

We conclude with an observation that string crossing number of $G$ can be defined in different ways analogously with the crossing number: the faithful string crossing number counts just the crossings among strings representing non-adjacent vertices of $G$, and the pair string crossing number counts just the pairs of non-adjacent vertices whose strings cross. The pair string crossing number actually counts the minimum number of edges that need to be added to $G$ to obtain a string graph containing $G$. The inequalities between these variants of string crossing number are obvious, but it is unclear whether there are any equalities.

Acknowledgements The authors are grateful to the referees for the enthusiasm expressed in their reports as well as for several valuable suggestions on how to improve the presentation by adding more prose to the paper.

\section{References}

1. Aigner, M., Ziegler, G.M.: Proofs from the Book. Springer, Berlin (2000)

2. Ajtai, M., Chvátal, V., Newborn, M.M., Szemerédi, E.: Crossing-free subgraphs. In: Rosa, A., Sabidussi, G., Turgeon, J. (eds.) Theory and Practice of Combinatorics: A Collection of Articles Honoring Anton Kotzig on the Occasion of His Sixtieth Birthday. Ann. Discrete Math., vol. 12, pp. 9-12. North-Holland, Amsterdam (1983)

3. Alexandrov, L., Djidjev, H.: Linear algorithms for partitioning embedded graphs of bounded genus. SIAM J. Discrete Math. 9, 129-150 (1996)

4. Benzer, S.: On the topology of the genetic fine structure. Proc. Natl. Akad. Sci. USA 45, 1607-1620 (1959)

5. Bokal, D.: On the crossing number of Cartesian products with trees. J. Graph Theory 56, 287-300 (2007)

6. Bokal, D., Fijavž, G., Mohar, B.: The minor crossing number. SIAM J. Discrete Math. 20, 344-356 (2006)

7. Bokal, D., Fijavž, G., Wood, D.R.: The minor crossing number of graphs with an excluded minor. Electron. J. Comb. 15, R4 (2008). http://www.combinatorics.org/Volume_15/Abstracts/v15i1r4.html

8. Bollobás, B., Leader, I.: Matchings and paths in the cube. Discrete Appl. Math. 75, 1-8 (1997)

9. Erdős, P., Guy, R.K.: Crossing number problems. Am. Math. Mon. 80, 52-58 (1973)

10. Garcia-Moreno, E., Salazar, G.: Bounding the crossing number of a graph in terms of the crossing number of a minor with small maximum degree. J. Graph Theory 36, 168-173 (2001)

11. Gilbert, J.R., Hutchinson, J.P., Tarjan, R.E.: A separator theorem for graphs of bounded genus. J. Algorithms 5, 391-407 (1984)

12. Houdré, C., Stoyanov, T.: Expansion and isoperimetric constants for product graphs. Combinatorica 26, 455-473 (2006)

13. Juarez, H.A., Salazar, G.: Optimal meshes of curves in the Klein bottle. J. Comb. Theory Ser. B 88, 185-188 (2003)

14. Kratochvíl, J.: String graphs I: the number of critical nonstring graphs is infinite. J. Comb. Theory Ser. B 52, 53-66 (1991)

15. Kratochvíl, J.: String graphs II: recognizing string graphs is NP-hard. J. Comb. Theory Ser. B 52, 67-78 (1991)

16. Kratochvíl, J., Matoušek, J.: String graphs requiring exponential representations. J. Comb. Theory Ser. B 53, 1-4 (1991)

17. Leighton, F.T.: Complexity Issues in VLSI. MIT Press, Cambridge (1983) 
18. Lipton, R.J., Tarjan, R.E.: A separator theorem for planar graphs. SIAM J. Appl. Math. 36, 177-189 (1979)

19. Mohar, B., Thomassen, C.: Graphs on Surfaces. Johns Hopkins University Press, Baltimore (2001)

20. Pach, J., Tóth, G.: Recognizing string graphs is decidable. Discrete Comput. Geom. 28, 593-606 (2002)

21. Pach, J., Shahrokhi, F., Szegedy, M.: Applications of crossing number. J. Graph Theory 22, 239-243 (1996)

22. Pach, J., Spencer, J., Tóth, G.: New bounds on crossing numbers. Discrete Comput. Geom. 28, 623644 (2000)

23. Pach, J., Radoičić, R., Tardos, G., Tóth, G.: Improving the crossing lemma by finding more crossings in sparse graphs. In: Snoeyink, J., Boissonnat, J.D. (eds.) Proceedings of the Twentieth Annual Symposium on Computational Geometry, pp. 68-75. ACM, New York (2004)

24. Richter, R.B., Thomassen, C.: Intersections of curve systems and the crossing number of $C_{5} \times C_{5}$. Discrete Comput. Geom. 13, 149-159 (1995)

25. Schaefer, M., Štefankovič, D.: Decidability of string graphs. J. Comput. Syst. Sci. 68, 319-334 (2004)

26. Schaefer, M., Sedgwick, E., Štefankovič, D.: String graphs in NP. J. Comput. Syst. Sci. 67, 365-380 (2003)

27. Shahrokhi, F., Sýkora, O., Székely, L.A., Vrt'o, I.: The crossing number of a graph on a compact 2-manifold. Adv. Math. 123, 105-119 (1996)

28. Sinden, F.W.: Topology of thin film RC circuits. Bell Syst. Tech. J. 45, 1639-1662 (1966)

29. Smith, W.D., Wormald, N.C.: Geometric separator theorems and applications. Manuscript (1998), 50 pp. http://www.math.uwaterloo.ca/ nwormald/papers/geomsep.ps.gz (2007)

30. Sýkora, O., Vrt'o, I.: Edge separators for graphs of bounded genus with applications. Theor. Comput. Sci. 112, 419-429 (1993)

31. Székely, L.A.: Crossing numbers and hard Erdős problems in discrete geometry. Comb. Probab. Comput. 6, 353-358 (1997)

32. Wada, K., Kawaguchi, K.: Optimal bounds of the crossing number and bisection width for generalized hypercube graphs. In: Proc. 16th Biennial Symposium on Communications, pp. 323-326 (1992) 